\title{
Frontières
}

\section{L’alchimie, ou la mort transmutée en immortalité}

\section{Christian Milat}

Volume 18, numéro 1, automne 2005

Hélas, célébrer la mort!

URI : https://id.erudit.org/iderudit/1074312ar

DOI : https://doi.org/10.7202/1074312ar

Aller au sommaire du numéro

Éditeur(s)

Université du Québec à Montréal

ISSN

1180-3479 (imprimé)

1916-0976 (numérique)

Découvrir la revue

Citer cet article

Milat, C. (2005). L'alchimie, ou la mort transmutée en immortalité. Frontières, 18(1), 33-38. https://doi.org/10.7202/1074312ar

\section{Résumé de l'article}

Selon la philosophie alchimique, l'état le plus élevé de l'être, celui de l'unité, est d'ordinaire dégradé en deux composantes de polarité opposée. Le travail de l'Adepte consiste à restaurer cette unité perdue lors d'opérations au cours desquelles la mort, manifestation de la perte de l'unité, devient le passage obligé vers le retour à l'unité et, partant, à l'immortalité. C'est donc dans la mesure où l'alchimie fait de la mort le prélude à tout état supérieur d'existence qu'elle la célèbre, mais cette célébration dépouille finalement la mort de toute portée funèbre.
Ce document est protégé par la loi sur le droit d'auteur. L'utilisation des services d'Érudit (y compris la reproduction) est assujettie à sa politique d'utilisation que vous pouvez consulter en ligne.

https://apropos.erudit.org/fr/usagers/politique-dutilisation/ 


\section{Résumé}

Selon la philosophie alchimique, l'état le plus élevé de l'être, celui de l'unité, est d'ordinaire dégradé en deux composantes de polarité opposée. Le travail de l'Adepte consiste à restaurer cette unité perdue lors d'opérations au cours desquelles la mort, manifestation de la perte de l'unité, devient le passage obligé vers le retour à l'unité et, partant, à l'immortalité. C'est donc dans la mesure où l'alchimie fait de la mort le prélude à tout état supérieur d'existence qu'elle la célèbre, mais cette célébration dépouille finalement la mort de toute portée funèbre.

Mots clés: alchimie - immortalité transmutation.

\section{Abstract}

According to alchemical philosophy, the highest state of being, unity, is ordinarily degraded into two components of opposite polarity. The Adept's work consists of restoring this lost unity through operations in which death, symbol of the loss of unity, becomes the mandatory pathway to the return to unity and thus immortality. Alchemy thus celebrates death by making it the prelude to any superior state of being, but this celebration removes death's morbid nature.

Keywords: alchemy - immortality transmutation.

$\begin{array}{lllllll}\text { A } & R & \mathrm{R} & \mathrm{I} & \mathrm{C} & \mathrm{L} & \mathrm{E}\end{array}$

\section{L'ALCHIMIE, \\ OU LA MORT TRANSMUTÉE EN IMMORTALITÉ}

\author{
Christian Milat, Ph.D., \\ professeur, Département des lettres françaises, \\ Université d'Ottawa
}

La pratique du Grand Euvre alchimique conduit le postulant à réaliser de multiples et subtiles opérations qui s'apparentent à des manipulations chimiques orientées notamment autour de ces deux opposés que sont le soufre et le mercure. À la suite de ces opérations aussi délicates que dangereuses - nombre d'apprentis sont morts victimes d'explosions ou d'intoxications -, l'«artiste» (Canseliet, 1965, t. I, p. 23) réalise l'argyropée, c'est-à-dire l'obtention de la Pierre blanche, capable de changer les métaux en argent, puis il atteint la chrysopée, qui lui permet d'obtenir la Pierre au rouge, poudre de projection qui, mise au contact de métaux vils comme le mercure, le plomb ou l'étain, aboutit à la transmutation de ceux-ci en or.

Parvenu à ce stade, l'Adepte n'a pourtant pas encore atteint son véritable objectif. En fait, la transmutation métallique lui permet essentiellement de vérifier si le produit qu'il vient d'obtenir est bien la poudre escomptée. En cas de succès, il continue d'effectuer quelques opérations qui, cette fois, lui font posséder la panacée ou médecine universelle, appelée également élixir de longue vie. L'ingestion périodique du précieux liquide guérit toutes les maladies, rend la jeunesse aux vieillards et prolonge indéfiniment la vie tout en procurant la connaissance absolue.
Certains Adeptes ont, semble-t-il, réussi le Grand Euvre sans pour autant avoir échappé à la mort. Ainsi, au XVII ${ }^{\mathrm{e}}$ siècle, l'Écossais Alexandre Sethon, dit le Cosmopolite, multiplia les transmutations de plomb en or avant de mourir sous la torture pour n'avoir point consenti à révéler son secret (voir Sadoul, 1970, p. 143-159). D'autres, plus chanceux, sont donnés comme ayant accompli l'ensemble de la tâche. Parmi eux figure, au XIV siècle, Nicolas Flamel, modeste écrivain public parisien qui, à la suite des transmutations de mercure en or qu'il aurait effectuées en 1382 (voir Sadoul, 1970, p. 96) avec l'aide de son épouse Pernelle, a acquis une fortune considérable; après avoir feint la maladie, le couple aurait organisé de fausses funérailles et vécu en Asie, et ce, au moins jusqu'au XVII ${ }^{\text {e }}$ siècle (voir Figuier, 1970, p. 245-246). La longévité alchimique expliquerait également l'existence, tout au long des $\mathrm{XVII}^{\mathrm{e}}$ et XVIII ${ }^{\mathrm{e}}$ siècles, d'un mystérieux Adepte qui a effectué de très nombreuses transmutations sous les identités successives d'Eyrénée Philalèthe, de Geraldi, de Lascaris et du comte de Saint-Germain (voir Sadoul, 1970, p. 179-237). Enfin, au XX $\mathrm{XX}^{\mathrm{e}}$ siècle, l'Adepte qui signa ses traités du pseudonyme de Fulcanelli aurait en 1922 donné à son disciple Eugène Canseliet les directives pour opérer une transmutation avant de disparaître, puis de reparaître en 1952 (voir Rivière, 1990, p. 29), dans la force de l'âge, alors qu'il avait 113 ans... 
Historiquement, si du moins on accorde crédit à ce qui n'est peut-être que légende, la pratique alchimique apparaît donc aller de pair avec une certaine longévité. Néanmoins, c'est l'immortalité - laquelle implique l'intervention réitérée de la mort - qui constitue l'un des objectifs fondamentaux des alchimistes. Pour tenter de comprendre de quelle(s) mort(s) il s'agit et comment celle(s)-ci s'insère(nt) dans le processus qui conduit à l'immortalité, il est indispensable d'examiner au préalable le support philosophique sur lequel s'articule le travail alchimique.

\section{UNE VÉRITÉ UNIVERSELLE ET ÉTERNELLE}

Dénommée Ars Magna, l'alchimie possède une composante pratique essentielle: «Celui [...] qui [...] ne veut labourer [c'est-àdire, en rapport avec l'étymologie latine, travailler] [...], celui-là doit être regardé comme le plus vaniteux des ignorants » (Fulcanelli, 1965, t. II, p. 197). Il reste cependant que ce travail, qui vise à posséder la Pierre philosophale, repose sur une véritable philosophie, pour peu que l'on n'entende pas par là le résultat, obligatoirement partiel, relatif et éphémère, d'une spéculation intellectuelle, mais qu'on lui donne son acception ancienne de connaissance absolue, objective et immuable. Du reste, si les alchimistes se nomment eux-mêmes «philosophes»(Canseliet, 1965, t. I, p. 14), ils entendent par là qu'ils sont «amateur[s] de la Vérité» (Canseliet, 1972, p. 7), d'une "Vérité éternelle, universelle et indivisible» (Canseliet, 1964a, p. 16). C'est également la raison pour laquelle ils s'appellent «fils de science»(Fulcanelli, 1964, p. 164), de la «Haute Science»(Alleau, 1953, p. 149), de «la science [...] des causes» (Fulcanelli, 1965, t. I, p. 79), d'une «science véritable et positive» (Fulcanelli, 1964, p. 223).

À cette vérité qui, éternelle, se situe hors du temps, il est impossible de fixer une origine, sauf à faire coïncider cette origine avec l'origine même du monde, origine divine, comme le fait Zozime de Panopolis, un alchimiste grec du IV siècle:

Les anciennes et saintes Écritures disent que certains anges, épris

d'amour pour les femmes, descendirent sur la terre, leur enseignèrent les œuvres de la nature; et à cause de cela ils furent chassés du ciel et condamnés à un exil perpétuel. De ce commerce naquit la race des géants. Le livre dans lequel ils enseignèrent les arts est appelé Chêma; de là le nom de Chêma appliqué à l'art par excellence.

(Hutin, 1966, p. 29)

Pour rester dans le domaine de la légende, notons que les Chinois se seraient adonnés à l'alchimie dès 4500 avant notre ère.

C'EST L'IMMORTALITÉ -

LAQUELLE IMPLIQUE L'INTERVENTION RÉITÉRÉE DE LA MORT -

QUI CONSTITUE L'UN DES OBJECTIFS FONDAMENTAUX DES ALCHIMISTES.

Tout au plus, s'agissant de l'alchimie occidentale, peut-on en trouver les sources dans l'Égypte hellénistique, où se mêlent en particulier les doctrines appartenant au néoplatonisme, à la gnose païenne et au gnosticisme chrétien. À Alexandrie, dès le $\mathrm{II}^{\mathrm{e}}$ siècle après Jésus-Christ, apparaissent ainsi les textes du Corpus Hermeticum attribués à Hermès Trismégiste, tantôt dieu, tantôt ancien roi, mais toujours considéré comme «le père de la Sagesse Occulte, le fondateur [...] de l'alchimie» (Anonyme, 1970, p. 149) - d'où l'épithète hermétique accolée à art pour désigner l'alchimie -, textes qui furent notamment traduits en français par Louis Ménard, condisciple et ami de Baudelaire (voir Milat, 1997, p. 571-588). Le IV ${ }^{\mathrm{e}}$ siècle est particulièrement fécond pour l'Art sacré alexandrin, avec notamment des alchimistes tels que Zozime et Marie la Juive, l'inventrice du bain-marie. Au $\mathrm{VI}^{\mathrm{e}}$ siècle, l'alchimie gagne le monde byzantin puis, au VII ${ }^{\mathrm{e}}$ siècle, le monde islamique. Ce sont ensuite les Arabes qui, via l'Espagne et à l'occasion des Croisades, introduisent l'alchimie en Occident, où elle se développe à compter du XII ${ }^{\mathrm{e}}$ siècle.

En dépit de sa diversité géographique et de sa longue histoire, mais peut-être en raison de la spécificité de ses origines et de ses transmissions, l'alchimie repose sur une philosophie dont les principes essentiels sont toujours restés les mêmes. Évidemment, certains concepts ont pu être introduits à telle ou telle époque par tel ou tel alchimiste mais, fondamentalement, le corps de doctrine n'a pas varié, de telle sorte qu'il est possible d'affirmer que «[1]es Philosophes Hermétiques [...] sont tous d'accord entre eux: pas un ne contredit les principes de l'autre. Celui qui écrivait il y a trente ans parle comme celui qui vivait il y a deux mille ans » (Pernety, 1991, t. I, p. 11).

Si l'ensemble des alchimistes partagent le même savoir et si celui-ci est exprimé au moyen d'un noyau lexical qui est commun à tous, chacun, en revanche, illustre concepts et opérations d'une façon qui lui est propre. Or, à cette liberté s'ajoute le fait que l'alchimie étant une "science [...] ésotérique»(Canseliet, 1965, t. I, p. 12), «secrète» (Fulcanelli, 1965, t. I, p. 151), la nature exacte de ses opérations comme les matériaux auxquels celles-ci s'appliquent ne sont jamais clairement identifiés, mais toujours cachés sous le «voile des allégories»(Canseliet, 1965, t. I, p. 12). D’où une difficulté de compréhension et, partant, la possibilité d'interprétations multiples.

Ainsi, bien que les traités alchimiques aient la plupart du temps recours, pour évoquer les opérations et les matériaux qu'ils décrivent, à des noms de métaux ainsi qu'à des termes de chimie, il n'est pas sûr que, pour parvenir à accéder aux promesses du Grand Euvre, il faille manipuler le matras ou le creuset. Comme le souligne un Adepte, l'étudiant ne doit pas s'arrêter au «sens littéral» (Fulcanelli, 1965, t. I, p. 314) et confondre l'alchimie avec «l'archimie [...] qui enseigne la transmutation des métaux» (Fulcanelli, 1965, t. I, p. 71). En réalité, le travail alchimique utilise des matériaux « qu'on ne saurait trouver qu'en soi-même» (Fulcanelli, 1965, t. II, p. 57). Les multiples acteurs de l'Ars Magna présentés dans les traités ou sur les gravures hermétiques correspondent donc en fait à l'illustration des différents états atteints par un seul initié travaillant sur lui-même, puisque "l'Un [...] se rapporte à la personne de 1'“artiste", dont l'unité est posée comme condition indispensable à l'accomplissement de l'œuvre » (Jung, 1970, p. 332). Ainsi, c'est au plus profond de l'individu que l'opus alchimique vise, au terme d' "une ascèse psychique et spirituelle» (Hutin, 1979, p. 192), à faire apparaître «un état particulier de la conscience» (Alleau, 1953, p. 36), voire à générer une "illumination de la conscience»(Alleau, 1953, p. 34), étant entendu qu'avec l'alchimie, "il ne s'agit pas de mysticisme, mais d'une science réelle, dans laquelle [l'accomplissement] n'a pas un sens moral, mais très concret nous dirions presque physique - au point d'éveiller éventuellement certains pouvoirs supranormaux»(Evola, 1988, p. 3), dont la possession "dote l'Adepte [...] de la vie éternelle, de l'infuse connaissance et des richesses temporelles, au sens le plus absolu de ces trois vocables et de leurs épithètes»(Canseliet, 1972, p. 67).

Étant donné que nombre de traités hermétiques font appel à un vocabulaire et à une iconographie appartenant à l'érotique ${ }^{1}$, certains ont vu dans la pratique alchimique une ascèse consistant, pour un homme et une femme, dans la maîtrise à deux de l'énergie sexuelle. Ainsi entendue, l'alchi- 


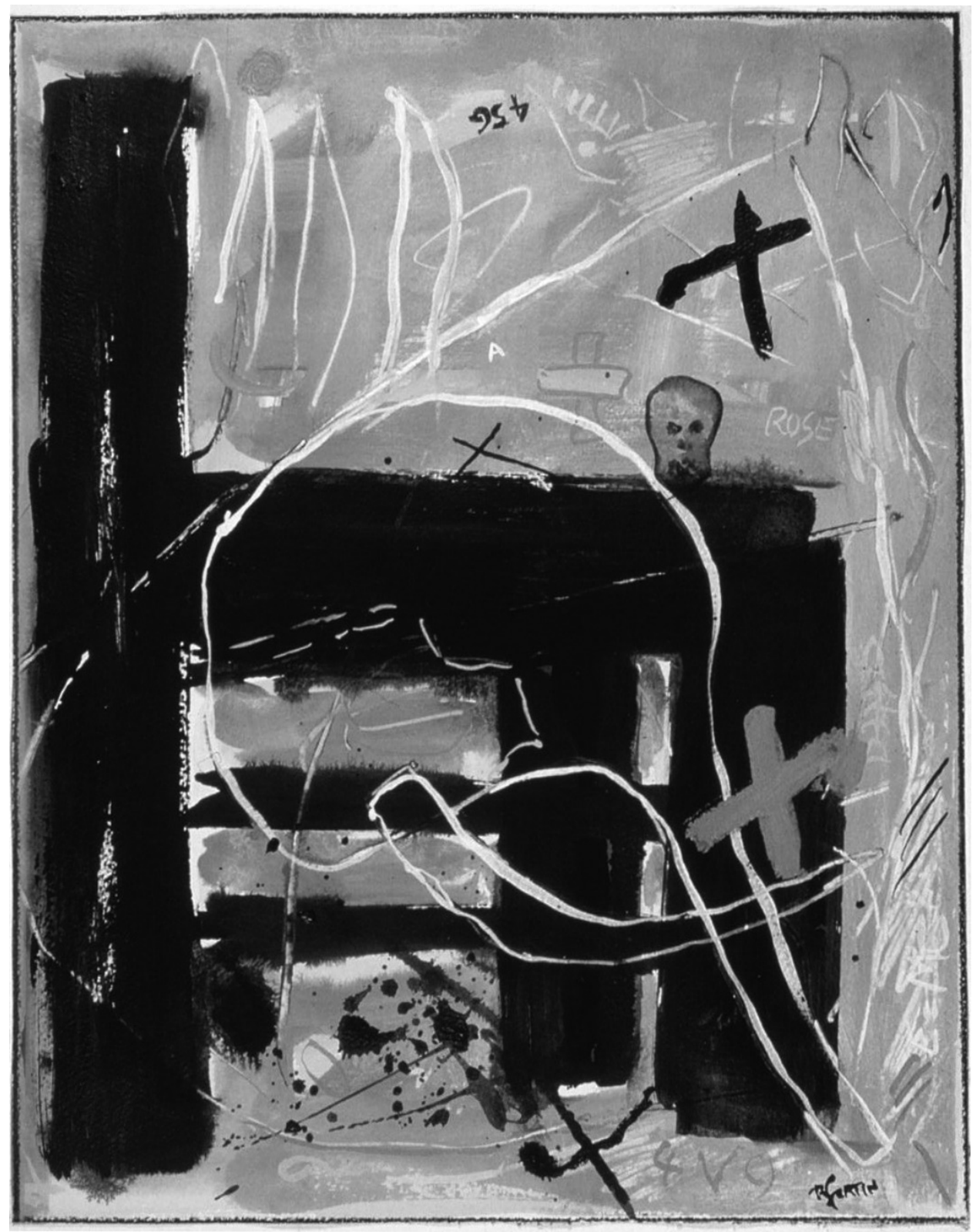

mie se confond avec le yoga tantrique. Selon celui-ci, s'entrelacent autour de la colonne vertébrale deux énergies subtiles qui sont opposées : idâ, qui correspond à la lune et à la déesse Çakti, et pingalâ, dont le caractère solaire est relié au principe illustré par le dieu Çiva. Il s'agit, notamment par le contrôle du souffle, de substituer à ces deux énergies, au cours de l'union sexuelle, l'énergie unique de la kundalini qui, elle, circule le long de l'axe de la colonne vertébrale, suspendant du même coup le temps et la mort (voir Evola, 1971, p. 224-226). L'objectif attribué ainsi à la sexualité s'apparente à la fonction impartie par Aristophane (voir Platon, 1950, p. 717-719) à Éros dans Le Banquet: permettre à l'homme et à la femme de recouvrer leur état androgynique primordial, auquel Zeus a mis fin en les coupant en deux pour les punir d'avoir tenté d'attaquer les dieux en escaladant le ciel.
Dans la philosophie taoïste et l'alchimie chinoise qui en découle, ces deux opposés sont respectivement désignés par le yin et par le yang. En effet, «le yang a la nature du Ciel et tout ce qui est actif, positif et masculin est yang, tandis que le yin a la nature de la Terre et tout ce qui est passif, négatif et féminin est yin» (Evola, 1968, p. 168). À ces deux principes antagonistes qui, au demeurant, ne sont pas sans évoquer les lois de la physique moderne ${ }^{2}$, sont associés tous les couples de contraires. C'est ainsi que s'explique "l'attribution au yin de la qualité froide, humide et obscure, au yang de la qualité sèche, claire, lumineuse» (Evola, 1968, p. 169). Par ailleurs, alors que «l'esprit est yang» (Evola, 1968, p. 168), au yin «est propre l'ombre, [...] en rapport avec les puissances élémentaires antérieures à la forme, qui chez l'être humain correspondent à l'inconscient» (Evola, 1968, p. 169). Enfin, «[t] ous les phénomènes, les formes, les êtres et les changements sont considérés à l'échelle de rencontres et de combinaisons variées du yang et du yin» (Evola, 1968, p. 168). C'est la raison pour laquelle «le pur yin et le pur yang» sont deux principes qui n'existent que dans l'absolu et qui sont toujours en fait entachés chacun d'une parcelle, plus ou moins grande, appartenant à son contraire, ainsi qu'il apparaît du reste dans le T'ai-Chi, symbole taoïste où, dans chacune des deux moitiés, noire et blanche, du dessin figure un petit disque de la couleur opposée (voir Rivière, 1990, p. 36-37).

Pour Jung, derrière la matière alchimique et les multiples formes de celleci, il faut comprendre «la psyché» (Jung, 1970, p. 62) et ses «manifestations », les deux opposés représentant l'inconscient et la conscience. Là encore, l'inconscient possède un "aspect chtonien et féminin » (Jung, 1970, p. 34); il figure «la moitié obscure de la personnalité, l'ombre » (Jung, 1970 , p. 45) et les «impulsions animales» (Jung, 1970, p. 192); c'est «un élément passif (patiens), réceptif, qui conçoit, ou une substance à laquelle il faut donner forme (alchimiquement informatio, le fait de donner une forme, impregnatio, fécondation)»(Jung, 1980, p. 66), tandis que la conscience "apparaît comme masculine, c'est-à-dire comme détermination active et action » et correspond "à l'agression». Toutefois, ce partage, selon Jung, doit être nuancé "puisque, selon la doctrine alchimique, chaque élément renferme son contraire "à l'intérieur" de lui-même» (Jung, 1980, p. 100). Ainsi, par exemple, le «Mercure, qui [...] personnifie l'inconscient, est essentiellement duplex, c'est une nature double paradoxale, un diable, une bête, et en même temps un remède salutaire» (Jung, 1980, p. 47). 
Se rapportant, dans l'ensemble de l'univers, indifféremment au plus petit et au plus grand, le principe de polarité, lequel «exprime la dualité propre à toute manifestation» (Rivière, 1990, p. 36), hisse l'alchimie au rang d'une philosophie, et notamment d'une métaphysique et d'une ontologie. Mais cette philosophie, nous l'avons vu, est en même temps une «philosophie expérimentale» (Fulcanelli, 1965, t. II, p. 75). Appliquant à l'homme, « appelé microcosme [...] parce qu'il offre en abrégé toutes les parties de l'univers» (Hutin, 1966, p. 63), les lois du macrocosme, les alchimistes revendiquent un art où le futur Adepte s'adonne à "un travail persévérant» (Fulcanelli, 1965, t. II, p. 133) grâce auquel l'alchimiste peut reconstituer l'unité idéale: «l'opus naît de l'Un et ramène à l'Un» (Jung, 1970, p. 377). Pour atteindre cet objectif, symbolisé notamment par l'élaboration de la Pierre philosophale ou par la naissance de l'Androgyne, l'étudiant doit effectuer toute une série d'opérations dont l'illustration fait indifféremment appel à la terminologie de la chimie ou au vocabulaire de l'érotique et dont la fin est la réalisation de la réunification des deux opposés sous l'effet d'un agent identifié au magnétisme ou au désir, la réalisation «du mariage, de l'union des deux principes, masculin (le Soufre) et féminin (le Mercure), [s'effectuant,] dans la cornue ou le creuset, par le moyen d'un troisième principe (le Sel), grâce auquel les "Noces chimiques" deviennent possibles " (Hutin, 1994, p. 73). Traditionnellement, ces opérations sont regroupées autour de trois phases «se développ[a]nt selon l'ordre invariable qui va du noir au rouge en passant par le blanc» (Fulcanelli, 1964, p. 108), ces trois couleurs n'étant pas utilisées pour identifier des matériaux distincts, mais pour signaler les «trois états successifs d'une même substance» (Fulcanelli, 1965, t. I, p. 279).

\section{L'CEUVRE AU NOIR}

Dans l'alchimie, dont la «dénomination principale [...] est Ars Regia, c'est-à-dire Art Royal»(Evola, 1988, p. 3), l'unité primordiale, laquelle constitue l'alpha et l'oméga de l'Euvre en ce sens qu'elle est donnée nécessairement à l'origine de toute chose ainsi que de tout être et qu'elle est l'objet dont la restauration représente l'objectif ultime de l'initié, est notamment présentée sous les traits du «roi » (Jung, 1970, p. 390). Réunissant en une seule personne les attributs tant masculins que féminins, ce roi est donc en même temps la reine puisqu'il joue le rôle de la mère, et c'est pourquoi il est souvent représenté sous la forme d'un roi dont l'unique couronne est posée sur deux têtes, l'une masculine, l'autre féminine, ces deux têtes étant étroitement imbriquées ${ }^{3}$. Parfois aussi, c'est la croix qui symbolise «l'"Homme Universel" » (Guénon, 1984, p. 25 et 27), c'est-à-dire «l'ensemble "AdamÈve" », l'entité androgyne précédant la chute, cette croix pouvant se substituer au signe $\mathrm{X}$, à la fois lettre majuscule, «marque de l'illumination» (Fulcanelli, 1965, t. I, p. 244), et chiffre romain, «nombre complet de l'Euvre». Parfois, enfin, l'unité est manifestée par «le ciel et la terre, qui étaient unis au commencement»(Guénon, 1994, p. 362).

Au cours de la phase initiale de l'opus, l'Euvre au Noir, appelé également «mortificatio, putrefactio, nigredo » (Eliade, 1977, p. 129), le "premier effet de l'“union occulte” »(Evola, 1968, p. 351) des opposés provoquée par le désir au sein de l'androgyne primordial aboutit à l'éclatement de celui-ci: le «Roi [...] meurt». En suscitant et en nourrissant le désir de l'autre, Éros a en effet posé l'existence d'un autre et, partant, il a entraîné la mort de l'unité. C'est d'ailleurs à cause de ce pouvoir mortifère d'Éros que «certaines divinités antiques [...] sont à la fois divinités de l'Amour et de la Mort» (Evola, 1988, p. 157).

Analogue au serpent biblique coupable de la chute, l'Éros alchimique est donc le responsable de la perte de l'unité primordiale: "[...] [L]a cause de la mort est l'amour [...]» (Hermès Trismégiste, 1945, t. I, p. 13), proclame la sagesse hermétique. L'union érotique du principe masculin et du principe féminin implique en effet l'individualisation préalable des opposés, lesquels étaient confondus dans l'état idéal, "l'essence de la totalité humaine» (Jung, 1970 , p. 25), que le psychanalyste suisse appelle le «soi». Éros est donc le feu, le soleil, lesquels constituent pour les alchimistes «l'unique agent des métamorphoses successives de la matière originelle, sujet et fondement du Magistère » (Fulcanelli, 1965, t, II, p. 198). Dans la figure correspondant à la quatrième des douze clefs de la philosophie alchimique (voir Valentin, 1956, p. 127), Éros est ce flambeau qui brille auprès d'un squelette qui se tient debout sur son cercueil.

La mort du roi «laisse apparaître une coloration bleu foncé ou noire, affectée au Corbeau, hiéroglyphe du caput mortuum de l'CEuvre. Tel est le signe et la première manifestation de la dissolution, de la séparation des éléments» (Fulcanelli, 1964, p. 198). En effet, la figure unitaire du roi est fragmentée $\mathrm{du}$ fait que sa composante consciente, sulfureuse, s'individualise en la double personne $\mathrm{du}$ mari/père et du fils, et que sa partie inconsciente, mercurielle, s'incarne indifféremment dans l'épouse ou dans la fille, selon qu'intervient l'un des deux éléments de l'unité primordiale ou le fruit de l'union disjonctive. Ainsi, l'union des principes masculin et féminin qui s'effectue au cours de la première étape de l'Euvre au Noir, loin de sceller leur conjonction, consomme en réalité leur irrémédiable disjonction.

S'ouvre alors la seconde étape de l'Euvre au Noir, au cours de laquelle le Soufre et le Mercure disjoints, "de nature et de tendances contraires, de complexion opposée» (Fulcanelli, 1965, t. II, p. 103), luttent «sous l'influence du feu» (Fulcanelli, 1965, t. I, p. 294) d'Éros. Chez le principe masculin, Éros engendre «une cruauté et une férocité qui est la contrepartie de la sensation transcendantale [...] de l'impossibilité de la substance féminine élémentaire d'être comblée. On veut "tuer" la femme occulte [...]» (Evola, 1968, p. 225). Point étonnant donc que les opposés mènent ce combat jusqu'à «la mort d'un des antagonistes» (Fulcanelli, 1965, t. II, p. 103), le Soufre «supplici[ant] [...] le Mercure» (Jung, 1971, p. 435), c'est-àdire le principe féminin, de manière que cette "torture" am[ène] [...] la "mort" » (Eliade, 1977, p. 129). Finalement, c'est «le mercure [...] [qui] meurt à la fin du premier stade de l'Guvre» (Fulcanelli, 1965, t. I, p. 293), «inaugur[ant] par le noir, sceau de sa mortification, la série chromatique du spectre philosophal».

Or, à l'issue du combat, le principe féminin a dû, avant de mourir, s'unir au principe masculin, et c'est au cours de cette union que l'inconscient mercuriel, vase alchimique, sorte «de matrice ou d'utérus » (Jung, 1970, p. 310), est fécondé par la conscience: il «reçoit la teinture ou semence du soufre, représentant le mâle» (Fulcanelli, 1965, t. I, p. 202). C'est donc nourri par le mercure en décomposition que le «sperme des philosophes[...][, qui est venu] féconder» (Jung, 1970, p. 509) la fiancée vierge de l'opus alchimique, va se développer jusqu'à devenir «le regius filius (fils du roi) » (Jung, 1970, p. 509), tant il est vrai que «[t]oute chair humaine ou animale ne peut produire nul accroissement ou propagation de son espèce, si ce n'est que ce soit d'abord par la putréfaction» (Valentin, 1956, p. 173).

\section{L'CEUVRE AU BLANC} ET L'CEUVRE AU ROUGE

À l'issue de l'Euvre au Noir, le Mercure mortifié, c'est-à-dire le principe féminin tué par la conscience masculine, est comparé à «une onde obscure» (Fulcanelli, 1965, t. I, p. 193) qui doit désormais «être lavée et blanchie». Étant donné que, dans l'Euvre alchimique, sans la «lumière céleste diffuse dans les ténèbres du corps, [...] rien ne se peut faire» (Fulcanelli, 1965, t. I, p. 174), c'est la conscience active qui se charge de cette tâche: "l'esprit [...] [va] travailler à l'épuration puis à la réfection de la substance modifiée et clarifiée avec l'aide du 
feu » (Fulcanelli, 1965, t. II, p. 84) de façon à obtenir «la pierre astrale, blanche, pesante, brillante comme pur argent»(Fulcanelli, 1965, t. I, p. 192), image de l'Euvre au Blanc. C'est ainsi qu'initialement symbole du «mercure» (Fulcanelli, 1965, t. I, p. 223), Diane deviendra l' «emblème spagyrique de l'argent et [...] sceau de la couleur blanche» (Fulcanelli, 1965, t. II, p. 23). À l'Euvre au Noir succédera ainsi l'Euvre au Blanc, «[d]e même que le jour, dans la Genèse, succède à la nuit, la lumière succède à l'obscurité» (Fulcanelli, 1964, p. 109).

Purifié par son opposé, le Mercure acquiert, à la suite de ce contact avec le Soufre, une "qualité mixte» (Fulcanelli, 1965, t. I, p. 296) qui en fait une préfiguration de l'unité à reconstituer. Toutefois, celle-ci n'étant pas encore parfaitement réalisée, l'harmonie est loin de régner entre les opposés. En effet, «redout[ant] l'intégration [...] à la conscience» (Jung, 1980, p. 135), le Mercure tire profit des attributs sulfureux dont il vient d'être doté pour affirmer sa supériorité sur son contraire, de telle sorte que «la Femme a le dessus sur l'Homme et le réduit à sa nature» (Evola, 1968, p. 197). En effet, "[1]'anima inconsciente est un être autoérotique, tout à fait incapable de relation, qui ne cherche rien d'autre que la prise de possession totale de l'individu, ce par quoi un homme se trouve féminisé d'étrange et pernicieuse manière» (Jung, 1980, p. 135). C'est pourquoi, au cours de cette "phase appelée régime de la Femme, des Eaux ou de la Lune, ou albedo » (Evola, 1968, p. 197), «l'homme [se] dissou[t] dans le principe qui lui est opposé».

C'est également pendant l'albedo que le Mercure, fécondé au moment de l'union sexuelle qui lui a été imposée au cours de l'Euvre au Noir, accouche de l'œuf philosophique dont la «coque [...] renferme le rebis philosophal» (Fulcanelli, 1964, p. 184), le «fils des philosophes» (Jung, 1970, p. 388), c'est-à-dire le composé androgyne dont l'éclosion a lieu durant l'épisode final du Grand Euvre.

L'Euvre au Rouge, ou rubedo, marque un revirement complet des rapports de force. En effet, la suprématie exercée pendant l'albedo par le principe féminin est cassée: "le Soufre et le Feu sont de nouveau actifs, le masculin $[\ldots]$ réagit sur la substance qui l'avait dissous, il prend le dessus sur le féminin [...], il lui transmet sa nature» (Evola, 1968, p. 352).

Armé de «l'épée, [...] hiéroglyphe du feu qui pénètre» (Fulcanelli, 1965, t. II, p. 15), c'est-à-dire pourvue de la puissance d'Éros, le Soufre de la conscience s'attaque donc au Mercure de l'inconscient, poursuivant et achevant sa lutte amorcée au cours de l'Euvre au Noir. Le principe féminin subit alors le «martyre spirituel» (Jung, 1971, p. 437), le «cruciatus (supplice, torture)» (Jung, 1971, p. 153) illustré dans les traités alchimiques par le "sacrifice du serpent» (Jung, 1971, p. 446), le «serpens mercurialis (serpent mercuriel) crucifié» (Jung, 1970, p. 505), lequel «caractéris[e] [le Christ] comme une personnification de l'inconscient» (Jung, 1971, p. 446) et témoigne d' «une victoire [de la conscience] sur l'inconscient».

Au milieu des sévices qu'il subit de la part de son opposé au cours de cette "penetratio» (Jung, 1971, p. 304), le Mercure « reste soumis au Soufre, car il est le serviteur et l'esclave, lequel, se laissant absorber, disparaît et se confond avec son maître » (Fulcanelli, 1965, t. II, p. 118). Cette assimilation s'effectue à l'occasion du «mariage des deux protagonistes»(Canseliet, 1972, p. 185), lequel prend la forme d'un «inceste [...] mère-fils» (Jung, 1970, p. 525), le fils, successeur du père, entreprenant de «tuer cette femme» (Evola, 1968, p. 276) avec laquelle il s'unit. Étant donné que cet «inceste symbolise l'union de l'être avec lui-même [...] [et représente] le degré d'union des semblables qui suit immédiatement l'idée originelle de l'autofécondation » (Jung, 1980, p. 77), l' «acte sacrificiel entrepris en vue de la transmutation alchimique $[\ldots][$, lequel réunit] sacrificateur et victime ${ }^{4}$ », ne met en réalité en relation qu'une seule et unique personne.

C'est ainsi que, sous «l'action violente du feu» (Fulcanelli, 1965, t. II, p. 75) érotique, l'union des deux opposés aboutit à la reconstitution de l'unité sous la forme d'un brasier où ont simultanément lieu «la destruction de[s] deux corps », à savoir le Mercure, mais aussi le Soufre, et l'éclosion de l'œuf philosophique, lequel a été expulsé au cours de l'Euvre au Blanc et d'où naît à présent le «Rebis, l'androgyne couronné» (Evola, 1968, p. 352) «héritier de l'énergie vitale et des qualités mixtionnées de ses parents défunts» (Fulcanelli, 1965, t. II, p. 75) puisqu'il «tient à la fois de la nature du soufre, son père, et de celle du mercure, sa mère» (Fulcanelli, 1965, t. II, p. 221): «[1]'enfant naît de leur mort et se nourrit de leurs cadavres» (Fulcanelli, 1965, t. II, p. 292), à l'image du «Phénix [... Hermaphrodite» (Bergerac, 1977, p. 465) qui renaît de ses cendres.

De fait, à l'issue de l'Euvre au Rouge, le principe masculin a réalisé l'unité en s'unissant à son opposé et en transférant à celuici, au cours de cette union et au moyen du feu d'Éros, sa propre nature, celle de l'élément feu. Le Grand Euvre est ainsi achevé avec cette «troisième opération [...] [qui] donne la Pierre philosophale» (Fulcanelli, 1964, p. 203), c'est-à-dire la «pierre qui porte le signe du soleil» (Fulcanelli, 1965, t. I, p. 177): l'Adepte, tel Jason, a trouvé la Toison d'or, dont la «fable [...] est une énigme complète du travail hermétique » (Fulcanelli, 1964, p. 194), et tel Hercule concluant ses travaux, il a cueilli «les pommes d'or du jardin des Hespérides» (Canseliet, 1964b, p. 145). L'or, "considéré comme [...] le "soleil des métaux"»(Guénon, 1994, p. 207), comme la «couleur royale» (Jung, 1970, p. 280) et, partant, le symbole de l'éternité, de l'immortalité, a transmuté le plomb, c'est-à-dire le métal vil évoqué par «Saturne ([...])» (Fulcanelli, 1965, t. II, p. 29), le «vieillard fabuleux [qui] dévore sa progéniture» (Fulcanelli, 1965, t. II, p. 190).

\section{LA RENAISSANCE PAR LA MORT}

C'est dire que le Grand Euvre constitue un travail sur le temps, et contre le temps. En effet, pour retrouver «l'état primordial» (Guénon, 1984, p. 64) où l'être humain possédait «le "sens de l'éternité", qui est aussi le "sens de l'unité" », le disciple du Grand Art entreprend de réunir les deux opposés en une atemporalité dynamique qu'exprime l'ouroboros, le serpent qui se mord la queue: "l'éternité, conçue sous l'aspect d'un “éternel retour”. Ce qui n'a ni fin ni commencement» (Van Lennep, 1966, p. 18).

Présente à la naissance du processus involutif puisque c'est elle qui sanctionne la fragmentation de l'unité originelle, cette mort en quelque sorte ontologique se perpétue par la suite en une mort ordinaire, qui frappe inéluctablement tous les individus oublieux de leur condition première et résignés à la dégradation duelle de leur identité. Pour les autres, ceux qui sont décidés à retrouver leur statut primordial et qui appliquent «l'axiome enjoignant de "tuer le vif pour ressusciter le mort" » (Trojani, 1996, p. 111), la mort devient l'instrument par lequel il est possible de provoquer la mort de la mort et, partant, d'accéder à l'immortalité. Ainsi, au cours de la seconde phase de l'Euvre au Noir, la mort tue en l'apprenti alchimiste la composante mercurielle, inconsciente, dont l'Euvre au Blanc régénère le cadavre. De la mort, enregistrée durant la phase de l'Euvre au Rouge, de cet élément ainsi que de son opposé conscient, soufré, naît l'androgyne, figure de l'unité et de l'immortalité recouvrées.

Ainsi, la philosophie et la pratique alchimiques, rejoignant là un précepte du travail des champs - d'où leur désignation «par la périphrase d'agriculture céleste» (Canseliet, 1964b, p. 155) -, comportent un enseignement fondamental: il n'y a pas de génération sans mort préalable, et rien ne meurt que pour renaître. C'est précisément dans la mesure où l'alchimie fait de la mort le passage obligé vers tout état supérieur d'existence qu'elle la célèbre, mais cette célébration dépouille finalement la mort de toute portée funèbre. 


\section{Bibliographie}

ALLEAU, R. (1953). Aspects de l'alchimie traditionnelle, Paris, Éditions de Minuit.

ANONYME (1970). Le Kybalion. Étude sur la philosophie hermétique de l'ancienne Égypte et de l'ancienne Grèce, Paris, Perthuis, [1917].

BERGERAC, C. de (1977). "L'Autre Monde», dans Euvres complètes, Paris, Librairie Belin, [1657 et 1662].

CANSELIET, E. (1964a). "Préface», dans FULCANELLI, Le Mystère des cathédrales et l'interprétation ésotérique des symboles hermétiques du grand œuvre, Paris, Jean-Jacques Pauvert, [1926].

CANSELIET, E. (1964b). Alchimie. Études diverses de Symbolisme hermétique et de pratique philosophale, Paris, Jean-Jacques Pauvert.

CANSELIET, E. (1965). «Préface», dans FULCANELLI, Les Demeures philosophales et le symbolisme hermétique dans ses rapports avec l'art sacré et l'ésotérisme du grand ouvre, Paris, Jean-Jacques Pauvert, [1930], t. I.

CANSELIET, E. (1972). L'Alchimie expliquée sur ses textes classiques, Paris, Jean-Jacques Pauvert.

CAPRA, F. (1979). Le Tao de la physique, Paris, Tchou [pour la trad.], [1975].

ELIADE, M. (1977). Forgerons et alchimistes, Paris, Flammarion, coll. "Champs», [1956].

EVOLA, J. (1968). Métaphysique du sexe, Paris, Payot [pour la trad.], [1958].

EVOLA, J. (1971). Le Yoga tantrique. Sa métaphysique, ses pratiques, Paris, Fayard [pour la trad.], coll. «Documents spirituels», [1949].

EVOLA, J. (1988). La Tradition hermétique. Les symboles et la doctrine de l'"Art Royal» hermétique, Paris, Éditions traditionnelles [pour la trad.], [1931].

FIGUIER, L. (1970). L'Alchimie et les alchimistes, Paris, Denoël, coll. «Bibliotheca Hermetica».

FLAMAND, É.-C. (1989). Érotique de l'alchimie, Paris, Le Courrier du livre.

FULCANELLI (1964). Le Mystère des cathédrales et l'interprétation ésotérique des symboles hermétiques du grand œuvre, Paris, Jean-Jacques Pauvert, [1926].

FULCANELLI (1965). Les Demeures philosophales et le symbolisme hermétique dans ses rapports avec l'art sacré et l'ésotérisme du grand œuvre, Paris, Jean-Jacques Pauvert, [1930], 2 vol.

GUÉNON, R. (1984). Le Symbolisme de la croix, Paris, Guy Trédaniel - Éditions Véga.

GUÉNON, R. (1994). Symboles de la science sacrée, Paris, Gallimard, [1962].

HERMÈS TRISMÉGISTE (1945). Corpus hermeticum, texte établi par A. D. Nock et traduit par A.-J. Festugière, Paris, Les Belles Lettres, 4 vol.

HUTIN, S. (1966). L'Alchimie, Paris, Presses universitaires de France, coll. «Que sais-je?».
HUTIN, S (1979). La Tradition alchimique, Saint Jean de Braye, Dangles.

HUTIN, S (1994). L'Amour magique, Bruxelles, Savoir pour Être.

JUNG, C. G. (1970). Psychologie et alchimie, Paris, Buchet/Chastel [pour la trad.], [1944].

JUNG, C. G. (1971). Les Racines de la conscience. Études sur l'archétype, Paris, Buchet/Chastel [pour la trad.], [1954].

JUNG, C. G. (1980). Psychologie du transfert, Paris, Albin Michel [pour la trad.], [1946].

MILAT, C. (1997). «Baudelaire, ou la dualité de l'Artiste à la poursuite de l'unité primordiale », Revue d'histoire littéraire de la France, vol. $97, \mathrm{n}^{\circ} 4$, p. 571-588.

PERNETY, A.-J. (1991). Les Fables égyptiennes et grecques, Paris, La Table d'Émeraude, [1786], 2 vol.

PLATON (1950). «Le Banquet, ou Del'amour», dans Euvres complètes, Paris, Gallimard, coll. «Bibliothèque de la Pléiade», t. I.

RIVIÈRE, P. (1990). L'Alchimie, science et mystique, Paris, De Vecchi.

SADOUL, J. (1970). Le Trésor des alchimistes, Paris, Denoël, coll. «En marge».

TROJANI, F. (1996). "Commentaires sur les dix-sept figures attribuées à Jean Conrad Barchusen», dans A. FAIVRE, Alchimie, Paris, Dervy, coll. "Cahiers de l'Hermétisme», p. 73-132.

VALENTIN, B. (1956). Les Douze clefs de la philosophie, Paris, Les Éditions de Minuit [pour la trad.], [1413].

VAN LENNEP, J. (1966). Art \& alchimie. Étude de l'iconographie hermétique et de ses influences, Bruxelles, Meddens.

\section{Notes}

1. Voir, par exemple, la gravure intitulée "Conjonctio sive Coïtus », où les deux amants royaux sont représentés, le soleil ou soufre philosophique pénétrant la lune ou mercure (Flamand, 1989, p. 121).

2. Ainsi, l'atome est par exemple formé d'un noyau de charge électrique positive et d'électrons chargés négativement. Voir notamment à ce sujet Capra (1979).

3. Voir, par exemple, la dixième gravure du traité d'alchimie intitulé le Rosarium Philosophorum (Jung, 1980, p. 178).

4. C. G. JUNG, Les Racines de la conscience. Études sur l'archétype, p. 151. 